OPEN ACCESS

Edited by:

Peter Hegyi,

University of Szeged, Hungary

Reviewed by:

Israr Ahmad,

University of Alabama at Birmingham,

United States

Gabor Varga,

Semmelweis University, Hungary

*Correspondence:

Roberta Squecco

roberta.squecco@unifi.it

Specialty section

This article was submitted to

Gastrointestinal Sciences,

a section of the journal

Frontiers in Physiology

Received: 24 August 2018

Accepted: 18 July 2019

Published: 06 August 2019

Citation:

Idrizaj E, Garella R, Castellini G,

Francini F, Ricca $V$, Baccari MC and

Squecco R (2019) Adiponectin Decreases Gastric Smooth Muscle

Cell Excitability in Mice.

Front. Physiol. 10:1000

doi: 10.3389/fphys.2019.01000

\section{Adiponectin Decreases Gastric Smooth Muscle Cell Excitability in Mice}

\author{
Eglantina Idrizaj ${ }^{1}$, Rachele Garella ${ }^{1}$, Giovanni Castellini ${ }^{2}$, Fabio Francini ${ }^{1}$, Valdo Ricca ${ }^{2}$, \\ Maria Caterina Baccari1 and Roberta Squecco ${ }^{1 *}$
}

1 Department of Experimental and Clinical Medicine, Section of Physiological Sciences, University of Florence, Florence, Italy,

${ }^{2}$ Psychiatric Unit, Department of Health Sciences, University of Florence, Florence, Italy

Some adipokines known to regulate food intake at a central level can also affect gastrointestinal motor responses. These are recognized to be peripheral signals able to influence feeding behavior as well. In this view, it has been recently observed that adiponectin (ADPN), which seems to have a role in sending satiety signals at the central nervous system level, actually affects the mechanical responses in gastric strips from mice. However, at present, there are no data in the literature about the electrophysiological effects of ADPN on gastric smooth muscle. To this aim, we achieved experiments on smooth muscle cells (SMCs) of gastric fundus to find out a possible action on SMC excitability and on membrane phenomena leading to the mechanical response. Experiments were made inserting a microelectrode in a single cell of a muscle strip of the gastric fundus excised from adult female mice. We found that ADPN was able to hyperpolarize the resting membrane potential, to enhance the delayed rectifier $\mathrm{K}^{+}$currents and to reduce the voltage-dependent $\mathrm{Ca}^{2+}$ currents. Our overall results suggest an inhibitory action of ADPN on gastric SMC excitation-contraction coupling. In conclusion, the depressant action of ADPN on the gastric SMC excitability, here reported for the first time, together with its well-known involvement in metabolism, might lead us to consider a possible contribution of ADPN also as a peripheral signal in the hunger-satiety cycle and thus in feeding behavior.

Keywords: adiponectin, gastric fundus, membrane properties, ion currents, satiety signals

\section{INTRODUCTION}

Adipokines are cytokines secreted by the white adipose tissue, able to influence a variety of physiological and pathophysiological processes through endocrine, paracrine, and autocrine mechanisms. ADPN, one of the most abundant adipokines secreted in the blood stream, regulates food intake by sending satiety signals at the central level, and exerts peripheral effects (Idrizaj et al., 2019). The observation that ADPN serum levels are correlated with body fat content and are

Abbreviations: $\mathrm{ADPN}$, adiponectin; $C_{\mathrm{m}}$, cell linear capacitance; $\mathrm{Ctrl}$, control; $G_{\mathrm{m}}$, membrane conductance; $G_{\mathrm{m}} / C_{\mathrm{m}}$, specific membrane conductance; HP, holding potential; $I_{a}$, current activation; $I_{\mathrm{Ca}}, \mathrm{Ca}^{2+}$ current; $I_{\mathrm{K}}$, voltage-dependent delayed rectifier $\mathrm{K}^{+}$currents; $k_{a}$, steepness factor of activation; $k_{\mathrm{h}}$, steepness factor of inactivation; RMP, resting membrane potential; SMC, smooth muscle cell; $V_{a}$, half-maximal activation voltage; $V_{\mathrm{h}}$, half-maximal inactivation voltage; $V_{\text {rev }}$, apparent reversal potential. 
lower in obese subjects (Bastard et al., 2006) has generated enormous interest within the scientific community (Henstridge and Febbraio, 2010). ADPN receptors, Adipo-R1 and AdipoR2, have been originally identified in the hypothalamus (Scherer et al., 1995). Previous studies (Hoyda and Ferguson, 2010) showed that ADPN acts at a central level, controlling neuronal excitability of the hypothalamic paraventricular nucleus through the modulation of different $\mathrm{K}^{+}$conductances and contributing to changes in membrane potential. Moreover, ADPN receptors have been found also in a variety of peripheral tissues (Yamauchi et al., 2003; Fasshauer et al., 2004; Kharroubi et al., 2004; Blüher et al., 2005; Liu et al., 2008; González et al., 2010; Hong et al., 2016) including the gastrointestinal tract (Idrizaj et al., 2018a). Acting through different signaling pathways, ADPN exerts antidiabetic, anti-inflammatory, antiatherogenic, and antiapoptotic effects (Idrizaj et al., 2019). Recently, other physiological roles of ADPN have emerged including the skeletal muscle sensitivity to this hormone (Krause et al., 2019), the prevention of cardiac dysfunctions (Francisco et al., 2016), and actions on the smooth muscle (AlSaif et al., 2015). Particularly, it has a proved vasorelaxant effect on vascular cells (Hong et al., 2016; Schinzari et al., 2017), and some of the mechanisms by which ADPN influences the contractile tone of small arteries have been clarified (Baylie et al., 2017). Besides vascular muscle activity, ADPN can influence that of the gastric one (Idrizaj et al., 2018a), but no effects of ADPN on the excitability of the SMCs of the gastrointestinal tract are reported at present. To this aim, we here intended to investigate the effect of this hormone on the bioelectric properties of SMC from the gastric fundus, focusing on the RMP, the ion currents responsible of the RMP control (Currò, 2014), and the voltage-dependent $I_{\mathrm{Ca}}$, mainly responsible for triggering the mechanical activity.

\section{MATERIALS AND METHODS}

The experimental procedure followed the European Community guidelines for animal care (DL 116/92, application of the European Communities Council Directive of 24 November 1986; 86/609/EEC) and was approved by the Committee for Animal Care and Experimental Use of the University of Florence in conformity with the Guide for the Care and Use of Laboratory Animals of the US National Institutes of Health (Idrizaj et al., 2018a,b). C57BL/6 (8-12 weeks old) female mice (Charles River, Lecco, Italy) were used (Squecco et al., 2013).

A muscular strip from the gastric fundus was pinned in a recording chamber (Squecco et al., 2013) bathed with a KrebsHenseleit solution (mM): $118 \mathrm{NaCl}, 4.7 \mathrm{KCl}, 1.2 \mathrm{MgSO}_{4}, 1.2$ $\mathrm{KH}_{2} \mathrm{PO}_{4}, 25 \mathrm{NaHCO}_{3}, 2.5 \mathrm{CaCl}_{2}$, and 10 glucose (pH 7.4). Intracellular recording was made by conventional microelectrode (resistance $=60-70 \mathrm{M} \Omega$ ) inserted in a cell of the longitudinal smooth muscle layer and filled with the following internal solution (mM): $130 \mathrm{KCl}, 10 \mathrm{NaH}_{2} \mathrm{PO}_{4}, 0.2 \mathrm{CaCl}_{2}, 1$ ethylenebis(oxyethylenenitrilo)tetraacetic acid (EGTA), $5 \mathrm{MgATP}$, and 10 4-(2-hydroxyethyl)-1-piperazineethanesulfonic acid (HEPES) ( $\mathrm{pH}$ 7.2), unless otherwise stated. We used the Krebs-Henseleit solution as Ctrl external solution to record RMP and passive properties of SMCs. In order to record $\mathrm{K}^{+}$current $\left(I_{\mathrm{K}}\right)$ we used the Krebs-Henseleit as external solution with specific channel blockers such as Nifedipine $(10 \mu \mathrm{M})$ for L-type $I_{\mathrm{Ca}}$, $\mathrm{BaCl}_{2}(0.4 \mathrm{mM})$ for eventual inward rectifier $\mathrm{K}^{+}$current, 4aminopyridine (4-AP, $2 \mathrm{mM}$ ) for eventual transient outward $\mathrm{K}^{+}$current (Castle and Slawsky, 1993; Crescioli et al., 2008). According to Idrizaj et al. (2018a), to record only $I_{\mathrm{Ca}}$ we used a high-TEA external solution $(\mathrm{mM}): 10 \mathrm{CaCl}_{2}, 145$ tetraethylammonium bromide, 10 HEPES, and a suitable filling pipette solution (mM): $150 \mathrm{CsBr}, 5 \mathrm{MgCl}_{2}, 10 \mathrm{EGTA}$, and 10 HEPES ( $\mathrm{pH}=7.2$ ). The current amplitude was normalized to cell capacitance, $C_{\mathrm{m}}$, to properly compare the currents recorded from cells of different size.

Recombinant full-length mouse ADPN was tested from $2 \times 10^{-11}$ up to $10^{-7} \mathrm{M}$. Heptanol $(1 \mathrm{mM})$ was consistently used to block gap junctional currents of the functional syncytium (Squecco et al., 2013). Drugs were from Sigma Chemical (St. Louis, MO, United States).

We recorded RMP of the SMCs before and after chemical stimulation in current clamp mode, with a stimulus waveform: $I=0$ pA (Squecco et al., 2015). The membrane passive properties were consistently estimated in voltage clamp starting from a $\mathrm{HP}$ of $-70 \mathrm{mV}$. $I_{\mathrm{K}}$ activation was elicited by 1 -s long voltage pulses ranging from -80 to $50 \mathrm{mV}$ applied in 10$\mathrm{mV}$ increments $(\mathrm{HP}=-60 \mathrm{mV})$. $I_{\mathrm{Ca}}$ kinetics was analyzed as in Idrizaj et al. (2018b). Mathematical analysis of data was performed by pClamp6 (Axon Instruments). Statistical analysis was done using Student's $t$-test or one-way ANOVA followed by Bonferroni's post hoc test when more than two groups of data were compared. $n$ represents the number of SMCs analyzed. Results are mean \pm SEM. $P \leq 0.05$ was considered significant unless otherwise specified.

\section{RESULTS}

\section{ADPN Hyperpolarizes the RMP of Gastric Fundus SMCs}

We first evaluated the effects of ADPN on the RMP of SMCs to assess its possible impact on cell excitability. Acute ADPN addition to the bath solution caused a hyperpolarization already appreciable at the lowest doses employed $\left(2 \times 10^{-11} \mathrm{M}\right)$ that reached the maximal value in about $3 \mathrm{~min}$ (Figure 1A). However, only starting from $2 \times 10^{-8} \mathrm{M}$ we observed a hyperpolarization statistically different compared to the RMP of the Ctrl cells. Higher doses did not cause further hyperpolarization (Figure 1B). This hyperpolarizing effect may indeed concur to hinder the SMC excitability (Squecco et al., 2015; Idrizaj et al., 2018b).

\section{Effects of ADPN on the Membrane Passive Properties of Gastric Fundus SMCs}

To estimate possible modifications of the SMC membrane passive properties, we first measured the $C_{\mathrm{m}}$ value in Ctrl condition and $10 \mathrm{~min}$ after the addition of ADPN to the external 


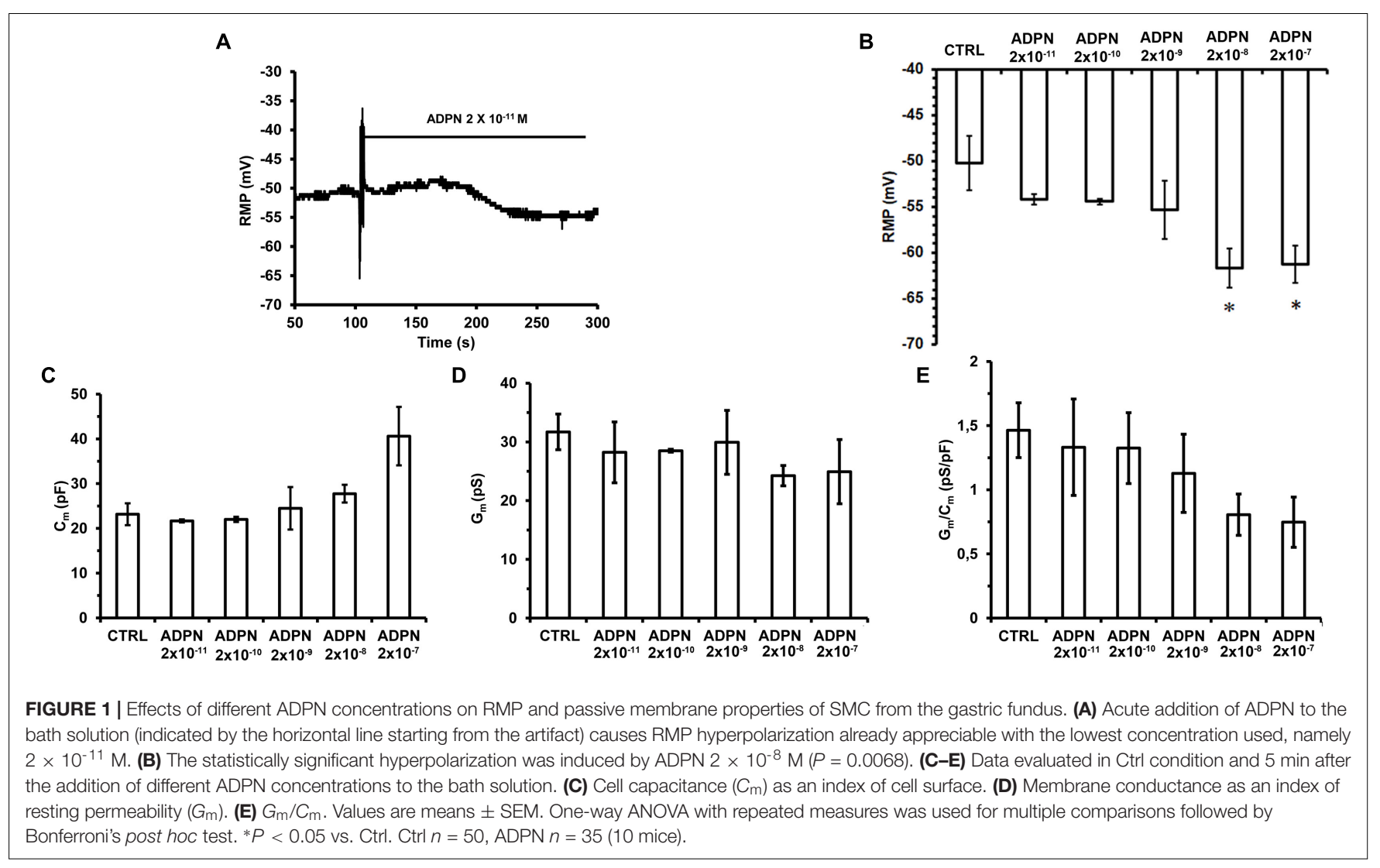

bath solution. Compared to the Ctrl values, ADPN induced a slight augmentation of $C_{\mathrm{m}}$ starting from a concentration of $2 \times 10^{-9} \mathrm{M}$, that became progressively higher as the dose increased, although not statistically significant for any concentration used (Figure 1C). The analysis of the $G_{\mathrm{m}}$ (Figure 1D) and of the specific conductance, $G_{\mathrm{m}} / C_{\mathrm{m}}$, in the presence of ADPN (Figure 1E) revealed a tendency to become smaller compared to the Ctrl values starting from $2 \times 10^{-8} \mathrm{M}$, indicating that ADPN scarcely affected the SMC membrane properties.

\section{ADPN Increases $I_{K}$ and Decreases $I_{\mathrm{Ca}}$ Amplitude in Gastric Fundus SMCs}

Trying to explain the observed membrane hyperpolarization, we tested the effects of ADPN on the main voltage-dependent $I_{\mathrm{K}}$ commonly supposed to Ctrl the RMP. Since the major effect of ADPN on the RMP was obtained at the concentration of $2 \times 10^{-8} \mathrm{M}$, we used this dose for all the following experiments. As expected, ADPN treatment increased $I_{\mathrm{K}}$ compared to Ctrl (Figures 2A-C) and this can undoubtedly contribute to membrane hyperpolarization.

Aiming to investigate if ADPN could affect the first steps of electro-mechanical coupling, we also evaluated its effect on $I_{\mathrm{Ca}}$. In Ctrl preparations, we constantly recorded inward current from the SMC resembling smooth muscular $I_{\mathrm{Ca}}$ (Figure 2D) with the $0-\mathrm{mV}$ step pulse evoking the maximal $I_{\mathrm{Ca}}$ amplitude. The acute addition of ADPN $\left(2 \times 10^{-8} \mathrm{M}\right)$ to the external bath solution decreased this current amplitude and caused a different voltage dependence. In fact, the maximal peak size was reached with the $10-\mathrm{mV}$ step pulse in the presence of ADPN (Figure 2E). The I$V$ curve analysis confirmed this general behavior (Figure 2F). We also performed the steady state analysis of the $I_{\mathrm{Ca}}$ activation and inactivation curves that were best-fitted by the Boltzmann function (Figure 2G): ADPN added to the bath solution strongly reduced the current size but did not affect the voltage dependence of its inactivation, whereas that of activation was positively shifted. The related Boltzmann parameters with the statistical significance are listed in Supplementary Table 1. These earliest results on $I_{\mathrm{Ca}}$ indicate that ADPN modulates $\mathrm{Ca}^{2+}$ influx altering the voltage-dependent channel kinetics in the gastric SMC.

\section{DISCUSSION}

Some adipokines that act at the central level to influence feeding behavior seem to affect gastrointestinal motor phenomena, which represent peripheral signals involved in the regulation of food intake (Duca and Covasa, 2012). In this view, leptin (Yarandi et al., 2011) and more recently ADPN appear to influence gastrointestinal motility in addition to their central actions. Particularly, ADPN is able to induce a decrease of the gastric mechanical activity in mice (Idrizaj et al., 2018a). The present results indicate for the first time that the hormone can influence the gastric SMCs' electrophysiological properties, which represent the first steps for the mechanical responses. Indeed, 


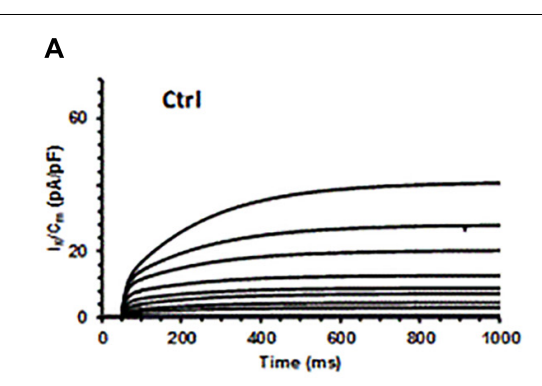

B

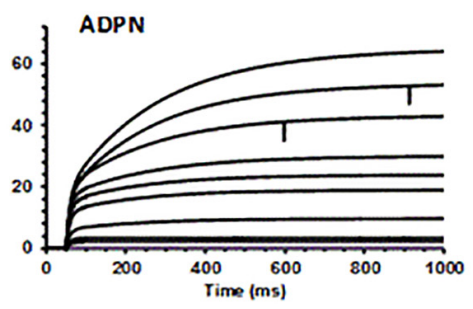

C

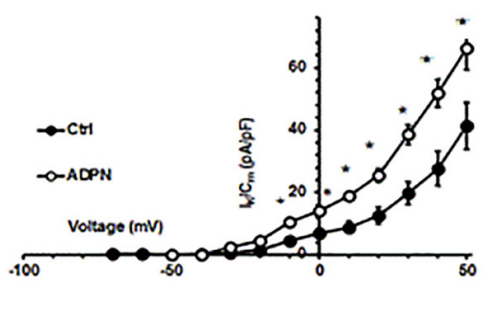

D

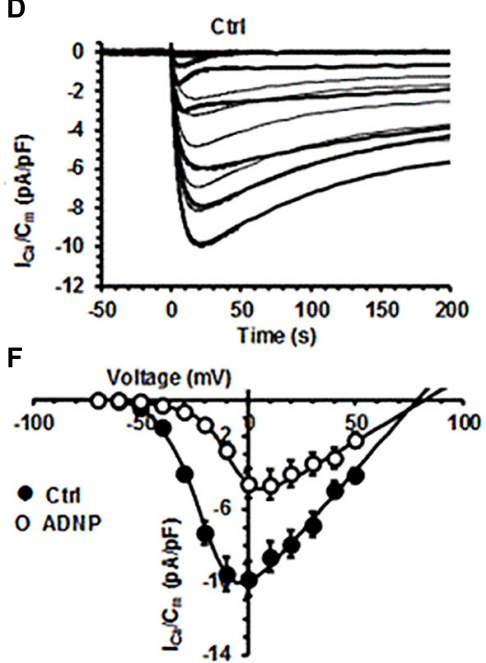

E

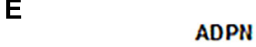

G
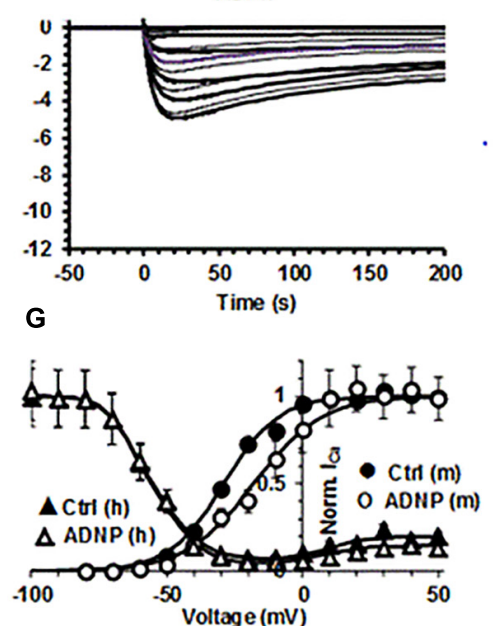

FIGURE 2 | Effects of ADPN on voltage-dependent $\mathrm{K}^{+}$current and Ca $_{\mathrm{Ca}}$ recorded in a SMC from the gastric fundus. (A,B) Representative total outward $\mathrm{K}^{+}$currents $\left(I_{\mathrm{K}}\right)$ recorded in Ctrl (A) and in the presence of ADPN $\left(2 \times 10^{-8} \mathrm{M}\right)$ (B), elicited by voltage steps from -80 to $50 \mathrm{mV}(\mathrm{HP}=-60 \mathrm{mV})$. (C) $/-V$ plots related to $/ \mathrm{K}$ in Ctrl (filled circles) and in the presence of ADPN (open circles). (D) Typical / $/$ a traces obtained in a Ctrl (D) and ADPN ( $\left.2 \times 10^{-8} \mathrm{M}\right)$ treated (E) SMCs in response to 4-S voltage pulses from -70 to $50 \mathrm{mV}(\mathrm{HP}=-80 \mathrm{mV})$, in 10-mV increments, in high-TEA bath solution. (F) $/-V$ plots related to /Ca in Ctrl (filled circles) and ADPN-treated SMCs (open circles). All the ADPN data points are statistically different $(P<0.05)$ to Ctrl over / Ca threshold. (G) Steady state activation $(\mathrm{m})$ and inactivation $(\mathrm{h})$ analysis of normalized / Ca: effect of ADPN (open circles) compared to Ctrl (filled circles) and lack of effects on inactivation (triangles). The continuous lines through the experimental data represent the fitted Boltzmann function. Note that, at positive potentials, the inactivation curve is U-shaped and decreased by ADPN. Current values are normalized to $C_{m}$. All of the data are mean values \pm SEM. Differences with $P<0.05$ were considered significant: ${ }^{*} P<0.05,{ }^{* *} P<0.01,{ }^{* * *} P<0.001$ (Student's t-test). Statistical significance is not depicted in the figure for clarity but is reported for the various Boltzmann parameters in Supplementary Table 1. Data are from 18 to 20 cells (four mice).

in keeping with our previous observation that ADPN induced gastric relaxation (Idrizaj et al., 2018a), we note that the hormone strongly influences SMCs' excitability by inducing membrane hyperpolarization. This effect can be determined, at least in part, by the here-observed tendency toward the reduction of $G_{\mathrm{m}}$, since this may hamper the aspecific entry of depolarizing ions, leading to a decreased SMC excitability. Moreover, we also noted that ADPN induces an increase of $I_{K}$, which is known to play an important role in RMP Ctrl. Although this effect was not extraordinarily broad, it may indeed contribute to the hyperpolarization. A more negative RMP definitely disturbs the related electromechanical coupling since a more intense stimulus than usual is required to activate high voltage thresholdoperated ionic channels (Dwyer et al., 2011; Idrizaj et al., 2018a). Accordingly, it became remarkable to study ADPN effect also on $I_{\mathrm{Ca}}$. In fact, this current represents a chief source for intracellular $\mathrm{Ca}^{2+}$ elevation useful for contractile activation and its eventual modifications may further affect the SMC mechanical activity.
Interestingly, we found that $\mathrm{ADPN}$ reduced $I_{\mathrm{Ca}}$ amplitude exerting an inhibitory effect on $\mathrm{Ca}^{2+}$ influx through voltagedependent $\mathrm{Ca}^{2+}$ channels, further supporting its influence in hindering the SMC electromechanical coupling.

However, our study raises several additional queries needing further investigation, such as the type of $\mathrm{K}^{+}$channel mostly involved ADPN effects, and the possible signaling pathways through which ADPN modulates the gastric SMC excitability. To answer these questions, further studies are in progress in our laboratory. Several signaling paths have been reported in relation to non-gastric smooth muscle and other targets for ADPN such as AMP-activated protein kinase (AMPK), peroxisome proliferative-activated receptor (PPAR)- $\alpha$ expression, ceramidase activity, and sphingosine 1 phosphate (S1P) formation (Botta et al., 2019; Kim and Park, 2019). This and some other previous reports dealing with NO signaling (Chen et al., 2003; Grossini et al., 2016; Nour-Eldine et al., 2016) will provide a useful background for our future studies. 


\section{CONCLUSION}

In conclusion, this preliminary study offers the first evidence that ADPN exerts a novel inhibitory function at the SMC plasma membrane level in gastric preparations that concurs to an actual weakened SMC excitability (Koh et al., 2012). ADPN seems to hinder the first steps of the excitation-contraction coupling, which is in perfect agreement with our previously published mechanical findings (Idrizaj et al., 2018a). Thus, ADPN seems to favor gastric muscle relaxation, which may lead to a consequent increase of organ capacity. Because gastric distension represents, from a physiological point of view, a peripheral satiety signal, we speculated that the here-observed peripheral effects are part of a control system designed to regulate food intake, which might concur to suppress feeding behavior. These observations provide a stimulating background to the challenging hypothesis that ADPN and/or its receptors could be a potential therapeutic tool in the treatment of obesity (Li et al., 2017) and eating disorders and, certainly, this issue deserves further investigation in a translational perspective.

\section{ETHICS STATEMENT}

This study was carried out in accordance with the recommendations of the European Community guidelines for animal care (DL 116/92, application of the European Communities Council Directive of 24 November 1986;

\section{REFERENCES}

AlSaif, S., Mumtaz, S., and Wray, S. (2015). A short review of adipokines, smooth muscle and uterine contractility. Life Sci. 125, 2-8. doi: 10.1016/j.lfs.2015.02.001

Bastard, J. P., Maachi, M., Lagathu, C., Kim, M. J., Caron, M., Vidal, H., et al. (2006). Recent advances in the relationship between obesity, inflammation, and insulin resistance. Eur. Cytokine Netw. 17, 4-12.

Baylie, R., Ahmed, M., Bonev, A. D., Hill-Eubanks, D. C., Heppner, T. J., Nelson, M. T., et al. (2017). Lack of direct effect of adiponectin on vascular smooth muscle cell $\mathrm{BKCa}$ channels or $\mathrm{Ca} 2+$ signaling in the regulation of small artery pressure-induced constriction. Physiol. Rep. 5:e13337. doi: 10.14814/ phy2.13337

Blüher, M., Fasshauer, M., Kralisch, S., Schön, M. R., Krohn, K., and Paschke, R. (2005). Regulation of adiponectin receptor R1 and R2 gene expression in adipocytes of C57BL/6 mice. Biochem. Biophys. Res. Commun. 329, 1127-1132. doi: 10.1016/j.bbrc.2005.02.081

Botta, A., Liu, Y., Wannaiampikul, S., Tungtrongchitr, R., Dadson, K., Park, T. S., et al. (2019). An adiponectin-S1P axis protects against lipid induced insulin resistance and cardiomyocyte cell death via reduction of oxidative stress. Nutr. Metab. 16:14.

Castle, N. A., and Slawsky, M. T. (1993). Characterization of 4-aminopyridine block of the transient outward $\mathrm{K}+$ current in adult rat ventricular myocytes.J. Pharmacol. Exp.Ther. 265, 1450-1459.

Chen, H., Montagnani, M., Funahashi, T., Shimomura, I., and Quon, M. J. (2003). Adiponectin stimulates production of nitric oxide in vascular endothelial cells. J. Biol. Chem. 278, 45021-45026. doi: 10.1074/jbc.m307878200

Crescioli, C., Squecco, R., Cosmi, L., Sottili, M., Gelmini, S., Borgogni, E., et al. (2008). Immunosuppression in cardiac graft rejection: a human in vitro model to study the potential use of new immunomodulatory drugs. Exp. Cell Res. 314, 1337-1350. doi: 10.1016/j.yexcr.2007.12.016

Currò, D. (2014). K+ channels as potential targets for the treatment of gastrointestinal motor disorders. Eur. J. Pharmacol. 733, 97-101. doi: 10.1016/j. ejphar.2014.03.049
86/609/EEC). The protocol was approved by the Committee for Animal Care and Experimental Use of the University of Florence in conformity with the Guide for the Care and Use of Laboratory Animals of the US National Institutes of Health.

\section{AUTHOR CONTRIBUTIONS}

EI and RS performed the electrophysiological experiments. EI, RG, FF, and RS analyzed the data. EI, RS, and FF prepared the figures. RS, MB, GC, and VR designed the research study. RS wrote the manuscript. RS, EI, MB, FF, RG, GC, and VR critically revised the manuscript.

\section{FUNDING}

This study was supported by funds from the University of Florence ATENEO RTD (CO 090101010107-SQUECCO_ RICTD16) to RS and GC, and Fondazione CRF (No. 2017.0777) to MB.

\section{SUPPLEMENTARY MATERIAL}

The Supplementary Material for this article can be found online at: https://www.frontiersin.org/articles/10.3389/fphys. 2019.01000/full\#supplementary-material

Duca, F. A., and Covasa, M. (2012). Current and emerging concepts on the role of peripheral signals in the control of food intake and development of obesity. $\mathrm{Br}$. J. Nutr. 108, 778-793. doi: 10.1017/S0007114512000529

Dwyer, L., Rhee, P. L., Lowe, V., Zheng, H., Peri, L., Ro, S., et al. (2011). Basally activated nonselective cation currents regulate the resting membrane potential in human and monkey colonic smooth muscle. Am. J. Physiol. Gastrointest. Liver Physiol. 301, 287-296. doi: 10.1152/ajpgi.00415.2010

Fasshauer, M., Kleinb, J., Kralischa, S., Kliera, M., Lössnera, U., Blüher, M., et al. (2004). Growth hormone is a positive regulator of adiponectin receptor 2 in 3T3-L1 adipocytes. FEBS Lett. 558, 27-32. doi: 10.1016/s0014-5793(03)01525-4

Francisco, C., Neves, J. S., Falcão-Pires, I., and Leite-Moreira, A. (2016). Can adiponectin help us to target diastolic dysfunction? Cardiovasc. Drugs Ther. 6, 635-644. doi: 10.1007/s10557-016-6694-x

González, C. R., Caminos, J. E., Gallego, R., Tovar, S., Vázquez, M. J., Garcés, M. F., et al. (2010). Adiponectin receptor 2 is regulated by nutritional status, leptin and pregnancy in a tissue-specific manner. Physiol. Behav. 99, 91-99. doi: 10.1016/j.physbeh.2009.10.015

Grossini, E., Farruggio, S., Qoqaiche, F., Raina, G., Camillo, L., Sigaudo, L., et al. (2016). Monomeric adiponectin modulates nitric oxide release and calcium movements in porcine aortic endothelial cells in normal/high glucose conditions. Life Sci. 161, 1-9. doi: 10.1016/j.lfs.2016.07.010

Henstridge, D. C., and Febbraio, M. A. (2010). Adiponectin sparks an interest in calcium. Cell Metab. 11, 447-449. doi: 10.1016/j.cmet.2010.05.007

Hong, K., Lee, S., Li, R., Yang, Y., Tanner, M. A., Wu, J., et al. (2016). Adiponectin receptor agonist, adiporon, causes vasorelaxation predominantly via a direct smooth muscle action. Microcirculation 23, 207-220. doi: 10.1111/micc. 12266

Hoyda, T. D., and Ferguson, A. V. (2010). Adiponectin modulates excitability of rat paraventricular nucleus neurons by differential modulation of potassium currents. Endocrinology 151, 3154-3162. doi: 10.1210/en.20091390

Idrizaj, E., Garella, R., Castellini, G., Mohr, H., Pellegata, N. S., Francini, F., et al. (2018a). Adiponectin affects the mechanical responses in strips from the mouse 
gastric fundus. World J. Gastroenterol. 24, 4028-4035. doi: 10.3748/wjg.v24.i35. 4028

Idrizaj, E., Garella, R., Francini, F., Squecco, R., and Baccari, M. C. (2018b). Relaxin influences ileal muscular activity through a dual signaling pathway in mice. World J. Gastroenterol. 24, 882-893. doi: 10.3748/wjg.v24.i8.882

Idrizaj, E., Garella, R., Squecco, R., and Baccari, M. C. (2019). Adipocytes-released Peptides involved in the control of gastrointestinal motility. Curr. Protein Pept. Sci. 20, 614-629. doi: 10.2174/1389203720666190121115356

Kharroubi, I., Ladrière, L., Cardozo, A. K., Dogusan, Z., Cnop, M., and Eizirik, D. L. (2004). Free fatty acids and cytokines induce pancreatic beta-cell apoptosis by different mechanisms: role of nuclear factor-kappaB and endoplasmic reticulum stress. Endocrinology 145, 5087-5096. doi: 10.1210/en.20040478

Kim, Y., and Park, C. W. (2019). Mechanisms of adiponectin action: implication of adiponectin receptor agonism in diabetic kidney disease. Int. J. Mol. Sci. 20:1782. doi: 10.3390/ijms20071782

Koh, S. D., Ward, S. M., and Sanders, K. M. (2012). Ionic conductances regulating the excitability of colonic smooth muscles. Neurogastroenterol. Motil. 24, 705718. doi: 10.1111/j.1365-2982.2012.01956.x

Krause, M. P., Milne, K. J., and Hawke, T. J. (2019). Adiponectin-consideration for its role in skeletal muscle health. Int. J. Mol. Sci. 20:E1528. doi: 10.3390/ ijms20071528

Li, F., Li, Y., Duan, Y., Hu, C. A., Tang, Y., and Yin, Y. (2017). Myokines and adipokines: involvement in the crosstalk between skeletal muscle and adipose tissue. Cytokine Growth Factor Rev. 33, 73-82. doi: 10.1016/j.cytogfr.2016. 10.003

Liu, B. H., Wang, Y. C., Wu, S. C., Mersmanna, H. J., Chenga, W. T., and Ding, S. T. (2008). Insulin regulates the expression of adiponectin and adiponectin receptors in porcine adipocytes. Domest. Anim. Endocrinol. 34, 352-359. doi: 10.1016/j.domaniend.2007.10.003

Nour-Eldine, W., Ghantous, C. M., Zibara, K., Dib, L., Issaa, H., Itani, H., et al. (2016). Adiponectin attenuates angiotensin ii-induced vascular smooth muscle cell remodeling through nitric oxide and the RhoA/ROCK pathway. Front. Pharmacol. 7:86. doi: 10.3389/fphar.2016.00086
Scherer, P. E., Williams, S., Fogliano, M., Baldini, G., and Lodish, H. F. (1995). A novel serum protein similar to C1q, produced exclusively in adipocytes. J. Biol. Chem. 270, 26746-26749. doi: 10.1074/jbc.270.45.26746

Schinzari, F., Tesauro, M., and Cardillo, C. (2017). Endothelial and perivascular adipose tissue abnormalities in obesity-related vascular dysfunction: novel targets for treatment. J. Cardiovasc. Pharmacol. 69, 360-368. doi: 10.1097/FJC. 0000000000000469

Squecco, R., Garella, R., Francini, F., and Baccari, M. C. (2013). Influence of obestatin on the gastric longitudinal smooth muscle from mice: mechanical and electrophysiological studies. Am. J. Physiol. Gastrointest. Liver Physiol. 305, 628-637. doi: 10.1152/ajpgi.00059.2013

Squecco, R., Garella, R., Idrizaj, E., Nistri, S., Francini, F., and Baccari, M. C. (2015). Relaxin affects smooth muscle biophysical properties and mechanical activity of the female mouse colon. Endocrinology 156, 4398-4410. doi: 10.1210/en.20151428

Yamauchi, T., Kamon, J., Ito, Y., Tsuchida, A., Yokomizo, T., Kita, S., et al. (2003). Cloning of adiponectin receptors that mediate antidiabetic metabolic effects. Nature 423, 762-769. doi: 10.1038/nature01705

Yarandi, S. S., Hebbar, G., Sauer, C. G., Cole, C. R., and Ziegler, T. R. (2011). Diverse roles of leptin in the gastrointestinal tract: modulation of motility, absorption, growth, and inflammation. Nutrition 27, 269-275. doi: 10.1016/j.nut.2010. 07.004

Conflict of Interest Statement: The authors declare that the research was conducted in the absence of any commercial or financial relationships that could be construed as a potential conflict of interest.

Copyright (C) 2019 Idrizaj, Garella, Castellini, Francini, Ricca, Baccari and Squecco. This is an open-access article distributed under the terms of the Creative Commons Attribution License (CC BY). The use, distribution or reproduction in other forums is permitted, provided the original author(s) and the copyright owner(s) are credited and that the original publication in this journal is cited, in accordance with accepted academic practice. No use, distribution or reproduction is permitted which does not comply with these terms. 